\title{
Fra-1 is upregulated in lung cancer tissues and inhibits the apoptosis of lung cancer cells by the P53 signaling pathway
}

\author{
GUANGWEI ZHONG ${ }^{1,2,3}$, XI CHEN $^{2}$, XIA FANG $^{2}$, DONGSHENG WANG $^{1}$, \\ MINGXUAN XIE ${ }^{2}$ and QIONG $\mathrm{CHEN}^{2}$ \\ ${ }^{1}$ Institute of Integrated Traditional Chinese and Western Medicine, ${ }^{2}$ Department of Geriatrics and ${ }^{3}$ International \\ Medical Center, Xiangya Hospital, Central South University, Changsha, Hunan 410008, P.R. China
}

Received July 31, 2015; Accepted September 23, 2015

DOI: $10.3892 /$ or.2015.4395

\begin{abstract}
Fos-related antigen-1 (Fra-1) is a member of the activator protein-1 transcription factor superfamily. It plays important roles in oncogenesis in various types of malignancies. Herein, we investigated the expression of Fra-1 in lung cancer tissues by qPCR, immunohistochemistry, and western blot technologies. The results showed that Fra-1 was overexpressed in the lung cancer tissues when compared with the level in the adjacent non-cancerous tissues. To explore the possible mechanism of Fra-1 in lung cancer, we elucidated the effect of Fra-1 on the apoptosis of lung cancer H460 cells, and found that the rate of cell apoptosis was decreased in the H460/Fra-1 cells compared with the H460 or H460/vector cells. Cell apoptosis is closely related with a reduction in mitochondrial membrane potential $(\Delta \Psi \mathrm{m})$ and an increase in intracellular reactive oxygen species (ROS) and calcium ion $\left(\mathrm{Ca}^{2+}\right)$ concentrations. Our results showed that overexpression of Fra-1 in the lung cancer H460 cells, led to an increase in $\Delta \Psi \mathrm{m}$ and and a decrease in intracellular $\mathrm{ROS}$ and $\mathrm{Ca}^{2+}$ concentrations. Furthermore, we found that Fra-1 was correlated with dysregulation of the P53 signaling pathway in lung cancer tissues in vitro. At the same time, we found that Fra-1 overexpression affected the expression of MDM2 and P53 in vivo. In summary, our results suggest that Fra-1 is upregu-
\end{abstract}

Correspondence to: Professor Qiong Chen, Department of Geriatrics, Xiangya Hospital, Central South University, Changsha, Hunan 410008, P.R. China

E-mail: qiongchxy@163.com

Abbreviations: Fra-1, Fos-related antigen-1; AP-1, activator protein-1; TP53, tumor protein p53; ROS, reactive oxygen species; DAB, 3,3'-diaminobenzidine tetrahydrochloride; FBS, fetal bovine serum; PI, propidium iodide; DCFH-DA, 2',7'-dichlorofluorescein diacetate; IHC, immunohistochemistry; GADPH, glyceraldehyde-3-phosphate dehydrogenase

Key words: Fra-1, lung cancer, P53, apoptosis lated in lung cancer tissues and functions by affecting the P53 signaling pathway in lung cancer.

\section{Introduction}

Lung cancer occurs and develops as a complicated result of an accumulation of various endogenous and exogenous effects $(1,2)$. Gene alterations participate in lung cancer genesis. ING5 inhibits cancer aggressiveness via preventing EMT and is a potential prognostic biomarker for lung cancer (3). Fibulin-5 is a metastasis suppressor in lung cancer by modulating the tumor microenvironment to suppress Wnt/ $\beta$-catenin signaling (4). Fra-1 enhances lung cancer epithelial cell motility and invasion by inducing the activity of MMPs, in particular MMP-2 and MMP-9, and EGFRactivated signaling (5). It has been shown that inactivation of tumor suppressor genes and activation of oncogenes play a significant role in carcinogenesis. However, the etiology of lung cancer remains poorly understood.

Fos-related antigen-1 (Fra-1, also known as FOSL1) is a member of the activator protein-1 (AP-1) transcription factor superfamily $(6,7)$. Fra-1 can positively regulate transcription. Fra-1 activity is regulated transcriptionally as well as post-translationally $(8,9)$. It is overexpressed in a variety of cancers, including lung, breast, colon and brain. High Fra-1 levels are associated with enhanced cell proliferation, migration, invasion and survival (6-9). Fra-1 plays a role in the progression and prognosis of non-small cell lung cancer (NSCLC) (10). Fra-1 can promote motility, invasion, and anchorage-independent growth of lung epithelial cells in vitro, but is insufficient for tumor formation (11). Although some evidence has been reported, the mechanism of Fra-1 in malignancy is not fully understood. Meanwhile, at present, few studies have investigated the relationship between Fra-1 and lung cancer. Thus, research on the effect and mechanism of Fra-1 in lung cancer is warranted.

TP53 is a crucial transcription factor and an important sensor of cellular stress under genotoxic, pathological, chemotoxic and even normal physiological conditions $(12,13)$. MDM2 is a negative regulator of TP53. Abnormalities in the TP53 gene and overexpression of MDM2 are commonly observed in malignancies. The MDM2-P53 feedback loop plays an important role in tumor progression $(14,15)$. Inactivation of the TP53 
tumor-suppressor gene is a common mechanism that cancer cells require to proliferate and escape pre-programmed cell death (16).

In this study, we examined the expression levels of Fra-1 in lung cancer tissues. At the same time, we studied the influence of Fra-1 on lung cancer and explored the possible mechanism.

\section{Materials and methods}

Cell culture. One identified general human lung cancer cell line, H460, was cultured in RPMI-1640 medium (Hyclone, Logan, UT, USA) supplemented with $10 \%$ fetal bovine serum (FBS; Gibco by Life Technologies ${ }^{\mathrm{TM}}$, Grand Island, NY, USA), $100 \mathrm{U} / \mathrm{ml}$ penicillin and $100 \mu \mathrm{g} / \mathrm{ml}$ streptomycin (GE Healthcare Life Sciences, Logan, UT, USA) at $37^{\circ} \mathrm{C}$ in the presence of $5 \% \mathrm{CO}_{2}$.

Patient samples. Ten participants were recruited at the Xiangya Hospital, Central South University (Changsha, Hunan, China). Consent forms were obtained from individual patients, and experimental protocols were approved by the Institutional Review Board of Xiangya Hospital. All subjects enrolled in the study were Chinese. All clinical and biological data were available for the samples (Table I). Lung cancer and corresponding non-tumor normal tissues were collected, and each biopsy sample was divided into two sections; one was submitted for routine histological diagnosis, and the remaining section was used for qPCR, immunohistochemistry and western blot experiments.

Total RNA extraction and quantitative real-time PCR analysis. Total RNA was extracted from the lung cancer and corresponding non-tumor normal tissues using TRIzol reagent, and cDNA synthesis was carried out using the RevertAid First Strand cDNA Synthesis kit (both from CWBio, Beijing, China) according to the manufacturer's recommendations. Quantitative real-time PCR (qRT-PCR) was carried out with GoTaq qPCR Master Mix (Promega, Madison, WI, USA). For detection of Fra-1 mRNA expression levels, GAPDH was amplified in parallel as an internal control. The sequences of the primers used for qPCR were as follows: Fra-1 forward, 5'-gcatgggctaaggatttgaa-3' and reverse, 5'-tcccaaatttagcctgttgg-3'; and GAPDH forward, 5'-cga ccactttgtcaagctca-3' and reverse, 5'-actgagtgtggcagggactc- 3 '. The expression of mRNA was assessed by evaluated threshold cycle (CT) values. The CT values were normalized with the expression levels of GAPDH, and the relative amount of mRNA specific to each of the target genes was calculated using the $2^{-\triangle \Delta C T}$ method (17-22). qPCR was carried out with the Bio-Rad CFK96 ${ }^{\mathrm{TM}}$ Real-Time system (Bio-Rad, Hercules, CA, USA). The data were analyzed by Bio-Rad CFK manager software (Bio-Rad). Expression of mRNA was assessed by the evaluated threshold cycle (CT) values and GAPDH was used as an internal control.

Immunohistochemistry (IHC) and evaluation of staining. Immunohistochemistry was conducted using the peroxidase anti-peroxidase technique following a microwave antigen retrieval procedure. The antibody for Fra-l was purchased
Table I. Characteristics of the lung cancer patients.

\begin{tabular}{ccll}
\hline Samples & $\begin{array}{c}\text { Age } \\
\text { (years) }\end{array}$ & Gender & \multicolumn{1}{c}{ Histological diagnosis } \\
\hline 1 & 58 & Male & Lung squamous cell cancer \\
2 & 56 & Male & Lung squamous cell cancer \\
3 & 62 & Male & Lung squamous cell cancer \\
4 & 58 & Male & Lung squamous cell cancer \\
5 & 67 & Male & Lung squamous cell cancer \\
6 & 55 & Male & Lung squamous cell cancer \\
7 & 65 & Male & Lung squamous cell cancer \\
8 & 72 & Female & Lung squamous cell cancer \\
9 & 57 & Male & Lung squamous cell cancer \\
10 & 56 & Male & Lung squamous cell cancer \\
\end{tabular}

from Boster Biotechnology (Wuhan, China). The antibody against Fra-1 (1:100) was overlaid on lung cancer and corresponding non-tumor normal tissue sections and incubated overnight at $4^{\circ} \mathrm{C}$. Secondary antibody incubation (Santa Cruz Biotechnology, Inc., Santa Cruz, CA, USA) was performed at room temperature for $30 \mathrm{~min}$. Color reaction was developed by using 3,3'-diaminobenzidine tetrahydrochloride (DAB) chromogen solution. All slides were counterstained with hematoxylin. Positive control slides were included in every experiment in addition to the internal positive controls. The specificity of the antibody was determined with a matched IgG isotype antibody as a negative control.

Sections were evaluated in a blinded manner by two investigators in an effort to provide a consensus on staining patterns by light microscopy (Olympus). Fra-1 staining was assessed according to the methods described by Hara and Okayasu (23) with minor modifications. Each case was rated according to a score that added a scale of intensity of staining to the area of staining. At least 10 high-power fields were chosen randomly, and $>1,000$ cells were counted for each section. The intensity of staining was graded on the following scale: 0 , no staining; 1+, mild staining; $2+$, moderate staining; $3+$, intense staining. The area of staining was evaluated as follows: 0 , no staining of cells in any microscopic fields; $1+,<30 \%$ of tissue stained positive; $2+$, between 30 and $60 \%$ stained positive; $3+,>60 \%$ stained positive. The minimum score when summed (extension + intensity) was, therefore, 0 , and the maximum, 6 . A combined staining score (extension + intensity) of $\leq 2$ was considered to be a negative staining (low staining); a score between 3 and 4 was considered to be moderate staining; whereas a score between 5 and 6 was considered to be strong staining. An optimal cut-off level was identified as follows: a staining index score of 0-2 was used to define tumors with negative expression and 3-7 indicated positive expression of these two proteins. Agreement between the two evaluators was $95 \%$, and all scoring discrepancies were resolved through discussion between the two evaluators.

Construction of the pEGFP-N1-Fra-1 vector. The coding region of the Fra- 1 gene was generated by PCR with the primer pair 5'-atactcgaatgaacctggccatcagcat-3' and 5'-gcggaattctc 
acagggacatgaaatccg-3'. PCR was performed under the following conditions: 1 cycle for $5 \mathrm{~min}$ at $94^{\circ} \mathrm{C} ; 30$ cycles for $45 \mathrm{sec}$ at $94^{\circ} \mathrm{C}, 45 \mathrm{sec}$ at $55^{\circ} \mathrm{C}$, and $90 \mathrm{sec}$ at $72^{\circ} \mathrm{C}$, and ended with $10 \mathrm{~min}$ at $72^{\circ} \mathrm{C}$. The fragments were cloned into the TA vector (Promega) and used to transform E. coli JM109 (Takara, Dalian, China). Following selection and propagation, the pure plasmid DNA was prepared by standard methods. The DNA fragments were removed from the TA vector by restriction enzyme digestion with $\mathrm{XhoI}$ and EcoR1 (Promega) to subclone into the pEGFP-N1 vector. The fusion sequences were verified by DNA sequencing using ABI 3730.

Cell transfection. Cell transfection was accomplished using Lipofectamine, according to the manufacturer's instructions (Invitrogen, USA). Cells $\left(2 \times 10^{5}\right)$ were plated into each well of a 6 -well plate $24 \mathrm{~h}$ prior to the transfection. For each transfection, $2 \mu \mathrm{g}$ of pEGFP-N1-Fra-1 plasmid and pEGFP-N1 vector plasmid were transfected into the $\mathrm{H} 460$ cells, respectively. The plasmids were diluted with $100 \mu$ of serum-free media, and $4 \mu \mathrm{l}$ Lipofectamine was added into $100 \mu \mathrm{l}$ serum-free media. The two solutions were combined, mixed gently and incubated at room temperature for $30 \mathrm{~min}$. Then $200 \mu \mathrm{l}$ of the mixture and $200 \mu \mathrm{l}$ of serum-free media were added into each well. The cells were then incubated at $37^{\circ} \mathrm{C}$ for $24 \mathrm{~h}$, followed by replacing the transfection media with fresh complete culture media. After an additional $48 \mathrm{~h}$ of culture, the cells were harvested for the following cell apoptosis, intracellular reactive oxygen species (ROS) measurement, and western blot experiments.

Effect of Fra-1 on lung cancer cell apoptosis. Cell apoptosis was analyzed by flow cytometric analysis using a MoFlo ${ }^{\mathrm{TM}}$ XDP High-Performance Cell Sorter (Beckman Coulter, Brea, CA, USA), propidium iodide (PI) and Hoechst 33342 double staining (Nanjing KeyGen Biotech Co., Ltd., Nanjing, China). Briefly, H460 cells were transfected transiently with pEGFP-N1-Fra-1 plasmid and the pEGFP-N1 vector plasmid, respectively. After $48 \mathrm{~h}$, the cells were collected in an Eppendorf tube and washed twice with PBS by centrifugation. The supernatants were discarded. To detect apoptosis, $500 \mu \mathrm{l}$ PBS, $5 \mu 1$ Hoechst 33342 and $5 \mu 1$ PI were added to each tube, and the contents of the tube were mixed in the dark at room temperature for $15 \mathrm{~min}$, followed by FCM testing (Beckman Coulter). Data were acquired and analyzed with Summit v5.2 software (Beckman Coulter).

Detection of mitochondrial membrane potential by JC-1. The impact of Fra-1 was measured by flow cytometry using the sensitive and relatively mitochondrion-specific lipophilic cationic probe fluorochrome JC-1. JC-1 accumulates to form $\mathrm{J}$-aggregates and emits red fluorescence in the mitochondria with a high membrane potential, yet dissociates into monomers and emits green fluorescence in those that lose cross-membrane electrochemical gradient. The cells were suspended in $1 \mathrm{ml}$ of warm staining buffer at $\sim 1 \times 10^{6}$ cells $/ \mathrm{ml}$ and incubated at $37^{\circ} \mathrm{C}$ for $5 \mathrm{~min}$. Then $1 \mu \mathrm{l}$ of $2 \mathrm{mM} \mathrm{JC}-1$ was added ( $2 \mu \mathrm{M}$ final concentration) and the cells were incubated at $37^{\circ} \mathrm{C}$ in $5 \% \mathrm{CO}_{2}$, for 15 to $30 \mathrm{~min}$. The cells were pelleted by centrifugation, resuspended by gently flicking the tubes, and $500 \mu \mathrm{l}$ PBS was added to each tube. Cells were analyzed with $\mathrm{MoFlo}^{\mathrm{TM}}$ XDP High-Performance Cell Sorter. Data were acquired and analyzed with Summit v5.2 software.

Intracellular ROS measurement. The production of intracellular ROS was measured by performing flow cytometry using the oxidation-sensitive probe, 2',7'-dichlorofluorescein diacetate (DCFH-DA; Applygen, Beijing, China). Briefly, $10 \mathrm{mM}$ DCFH-DA stock solution (in methanol) was diluted 4,000-fold in cell culture medium without serum or other additives to yield a 2.5-mM working solution. After exposure of human umbilical vein endothelial cells (HUVECs) to silica nanoparticles for 3 and $24 \mathrm{~h}$, respectively, the cells in 6-well plates were washed twice with PBS and incubated in $2 \mathrm{ml}$ working solution of DCFH-DA at $37^{\circ} \mathrm{C}$ in the dark for $30 \mathrm{~min}$. Then the cells were washed twice with cold PBS and resuspended in the PBS for analysis of intracellular ROS by FACS (Beckman Coulter).

Intracellular calcium ion $\left(\mathrm{Ca}^{2+}\right)$ concentration assay. Intracellular $\mathrm{Ca}^{2+}$ concentration was measured by means of the fluorescent $\mathrm{Ca}^{2+}$ chelator Fura-2 AM, which permeates into cells where it is cut into Fura-2, resorting within cells. Fura-2 combines with intracellular $\mathrm{Ca}^{2+}$ to form a fluorescent compound, whose fluorescent intensity was determined at an excitation wavelength of $340 \mathrm{~nm}$ and an emission wavelength of $510 \mathrm{~nm}$ in FACS (Beckman Coulter). After treatment, the cells were harvested and rinsed with PBS. The harvested cells were suspended in PBS and incubated with $5 \mu \mathrm{M}$ Fura-2 AM for $60 \mathrm{~min}$ at $37^{\circ} \mathrm{C}$. During the session of incubation with Fura-2 AM, the cell cultures were mildly shaken at intervals of $10 \mathrm{~min}$ aimed to facilitate the combination of Fura- 2 and $\mathrm{Ca}^{2+}$ to form the fluorescent compound. Then, the cells were washed twice and resuspended in PBS for FACS measurement (Beckman Coulter). Data were acquired and analyzed with Summit v5.2 software.

Western blot analysis. The lung cancer tissues, corresponding non-tumor normal tissues and $\mathrm{H} 460$ cells were lysed in RIPA buffer (CWBio), and total protein concentration was determined using the Pierce ${ }^{\circledR}$ BCA protein assay kit (Thermo Scientific, Inc., Rockford, IL, USA). Extracts containing $50 \mu \mathrm{g}$ of proteins were separated on $10 \%$ SDS-PAGE gels and electroblotted onto nitrocellulose membranes (Hyclone Laboratories). The membranes were inhibited using Tris-buffered saline/Tween-20 (25 mM Tris-HCl, $150 \mathrm{mM}$ $\mathrm{NaCl}$, pH 7.5 and $0.05 \%$ Tween-20) containing 5\% non-fat milk followed by overnight incubation at $4{ }^{\circ} \mathrm{C}$ with the primary antibodies (rabbit anti-MDM2 antibody, 1:200 and rabbit anti-P53 antibody, 1:200; Boster Biotechnology). Following three washes, the membranes were incubated with horseradish peroxidase-conjugated secondary antibodies (catalog no. sc-2491, dilution 1:5,000; Santa Cruz Biotechnology, Inc.) and the specific signals were visualized using an ECL detection system. The anti-GAPDH antibody (1:3,000; Santa Cruz Biotechnology, Inc.) was used as a loading control.

Statistical analysis. Differences in non-parametric variables were analyzed by the Mann-Whitney U test. Differences in the quantitative variables between groups were analyzed by Student's t-test using SPSS 11.0 program (SPSS, Chicago, IL, 
Table II. Assessment of the mRNA expression levels of Fra-1 in lung cancer and adjacent non-cancerous tissues by qPCR.

\begin{tabular}{llcccccc}
\hline Gene & \multicolumn{1}{c}{ Sample } & $\mathrm{n}$ & $\begin{array}{c}\text { Fra-1 CT } \\
(\text { mean } \pm \text { SD })\end{array}$ & $\begin{array}{c}\text { GAPDH CT } \\
(\text { mean } \pm \text { SD })\end{array}$ & $\begin{array}{c}\Delta C T \\
(\text { mean } \pm \text { SD })\end{array}$ & $\begin{array}{c}\Delta \Delta \text { CT } \\
(\text { mean } \pm \text { SD })\end{array}$ & Fold $^{\text {a }}$ \\
\hline Fra-1 & Lung cancer & 10 & $26.15 \pm 1.14$ & $19.24 \pm 0.64$ & $6.91 \pm 0.43$ & $-1.91 \pm 0.42$ & 3.76 \\
& Non-cancerous tissues & 10 & $28.23 \pm 1.17$ & $19.41 \pm 0.73$ & $8.82 \pm 0.39$ & & $(2.62-4.99)$ \\
\hline
\end{tabular}

${ }^{\mathrm{a}}$ Mean fold-change in expression of the target gene, Fra-1, relative to the internal control gene, GAPDH, was calculated using the $2^{-\Delta \triangle \mathrm{CT}}$ equation previously adopted by Livak et al (29): $\Delta \Delta \mathrm{CT}=\left(\mathrm{CT}_{\mathrm{Target}}-\mathrm{CT}_{\mathrm{GAPDH}}\right)$ lung cancer - $\left(\mathrm{CT}_{\mathrm{Target}}-\mathrm{CT}_{\mathrm{GAPDH}}\right)$ control. At least three replicates of each reaction were performed. $\mathrm{CT}$, threshold cycle; qPCR, quantitative polymerase chain reaction.

A

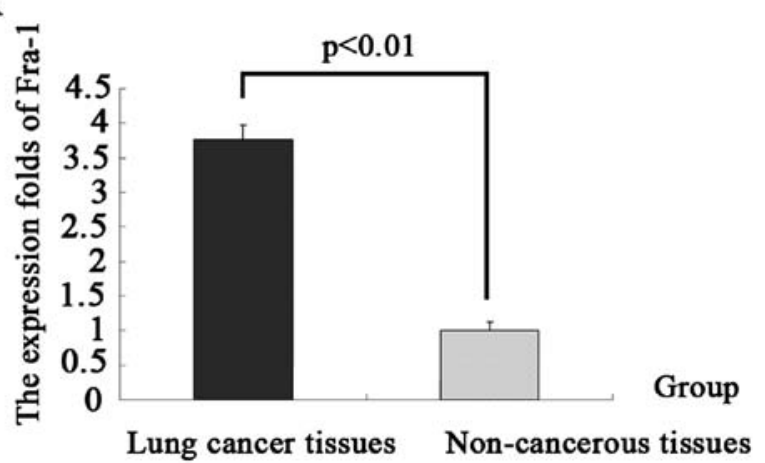

B

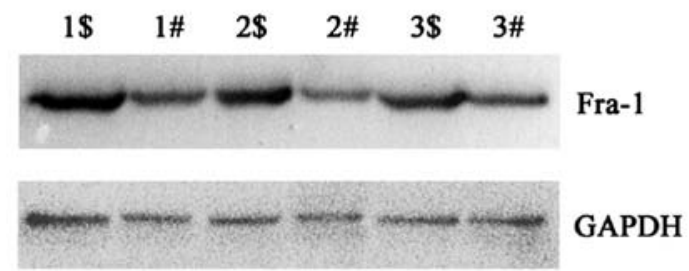

Figure 1. Detection of expression levels of Fra-1 in the lung cancer tissues and the adjacent non-cancerous tissues by qPCR and western blot analysis. (A) qPCR was performed to validate the expression of Fra-1 in the lung cancer and the adjacent non-cancerous tissues. GAPDH was used as an internal control and for normalization of the data. The mRNA expression level of Fra-1 was significantly upregulated in the lung cancer tissues $(n=10)$ compared with the level in the adjacent non-cancerous tissues $(n=10)$. (B) Tissues (1-3) which were used for the detection of mRNA expression levels by qPCR were selected to detect the expression levels of Fra-1 protein by western blot analysis. 1\$-3\$, lung cancer samples; $1 \#-3 \#$, adjacent non-cancerous tissues. Data are representative of three independent experiments.

USA). A probability value (p) $<0.05$ was considered to indicate a statistically significant result.

\section{Results}

Fra-1 expression is upregulated in lung cancer tissues. To detect the mRNA expression levels of the Fra-1 molecule in lung cancer and the adjacent non-cancerous tissues, 10 samples of each were selected to perform qPCR of the Fra-1 gene. The data were analyzed using the $2^{-\Delta \Delta \mathrm{CT}}$ method, and the fold change in the expression of this gene relative to the internal control gene, GAPDH, was analyzed. The expression of the Fra-1 gene was higher in the lung cancer samples compared with the adjacent non-cancerous tissues and the normalized
Fra-1 gene expression in lung cancer was upregulated by 3.76-fold ( $\mathrm{p}=0.0094<0.01)$ (Table II, Fig. 1A).

To determine whether the Fra-1 gene is expressed at a higher level in lung cancer compared with adjacent non-cancerous tissues, the protein expression levels of Fra-1 were further examined by western blot analysis in 1 to 3 samples (Fig. 1B). In comparison with the adjacent non-cancerous tissues, the expression level was identified to be higher in the lung cancer tissues, which corresponded with the qPCR results.

To confirm the pattern of Fra-1 in lung cancer, immunohistochemistry (IHC) was carried out with antibodies against the Fra-1 protein in lung cancer and the adjacent non-cancerous tissues. Fra-1 was identified as being differentially expressed between lung cancer tissues vs. the adjacent non-cancerous tissues. IHC showed a similar pattern in protein expression with the western blot results. A total of $50.09 \%$ (13/22) of lung cancer tissues had high Fra-1 expression and 13.64\% (3/22) of the adjacent non-cancerous tissues had a high score. The distribution of low scores was 9.09\% (2/22) and 63.64\% (14/22) in the lung cancer and the adjacent non-cancerous tissues, respectively $(\mathrm{p}=0.012<0.05)$ (Table III). This corresponded with the qPCR results.

Fra-1 inhibits the apoptosis of lung cancer cells in vitro. We found that Fra-1 was highly expressed in the lung cancer tissues by qPCR, western blot and IHC technologies. To elucidate the function of Fra-1 in the apoptosis of lung cancer cells, the lung cancer cell line, H460, was transfected with the plasmid pEGFP-N1/Fra-1 or the control vector. After determining Fra-1 protein by qPCR technologies, we performed a Hoechst 33342/PI double-staining experiment to test the rate of apoptosis in the H460, H460/vector, and H460/Fra-1 cells. A considerable decrease in apoptotic cells was observed in the H460/Fra-1 cells $(6.29 \pm 0.41 \%)$ compared with the $\mathrm{H} 460 /$ vector $(11.73 \pm 0.85 \%)$ and $\mathrm{H} 460$ cells $(12.04 \pm 0.92 \%)$ (Fig. 2A).

Fra-1 affects mitochondrial membrane potential ( $\Delta \Psi m)$, ROS and $\mathrm{Ca}^{2+}$ concentrations in lung cancer cells in vitro. Cell apoptosis is closely related with a reduction in $\Delta \Psi \mathrm{m}$ and an increase in intracellular $\mathrm{ROS}$ and $\mathrm{Ca}^{2+}$ concentrations. Thus, we tested the influence of Fra-1 on these three parameters. Our results showed that overexpression of Fra-1 in lung cancer $H 460$ cells, led to an increase in $\Delta \Psi_{\mathrm{m}}$ and inhibited cell apoptosis (Fig. 2B). ROS results showed that overexpression of Fra-1 in lung cancer H460 cells, led to a decrease in 
Table III. Difference in Fra-1 expression between the lung cancer and the adjacent non cancerous tissues.

\begin{tabular}{|c|c|c|c|c|c|}
\hline & \multirow[b]{2}{*}{$\mathrm{n}$} & \multicolumn{3}{|c|}{ Score } & \multirow[b]{2}{*}{ P-value } \\
\hline & & Low $(0-2)$ & Moderate (3-4) & High (5-6) & \\
\hline Lung cancer & 22 & $2(9.09 \%)$ & $7(31.82 \%)$ & $13(50.09 \%)$ & $=0.012<0.05$ \\
\hline Non-cancerous tissues & 22 & $14(63.64 \%)$ & $5(22.73 \%)$ & $3(13.64 \%)$ & \\
\hline
\end{tabular}

$\mathrm{p}<0.05$ by Mann-Whitney $\mathrm{U}$ test.
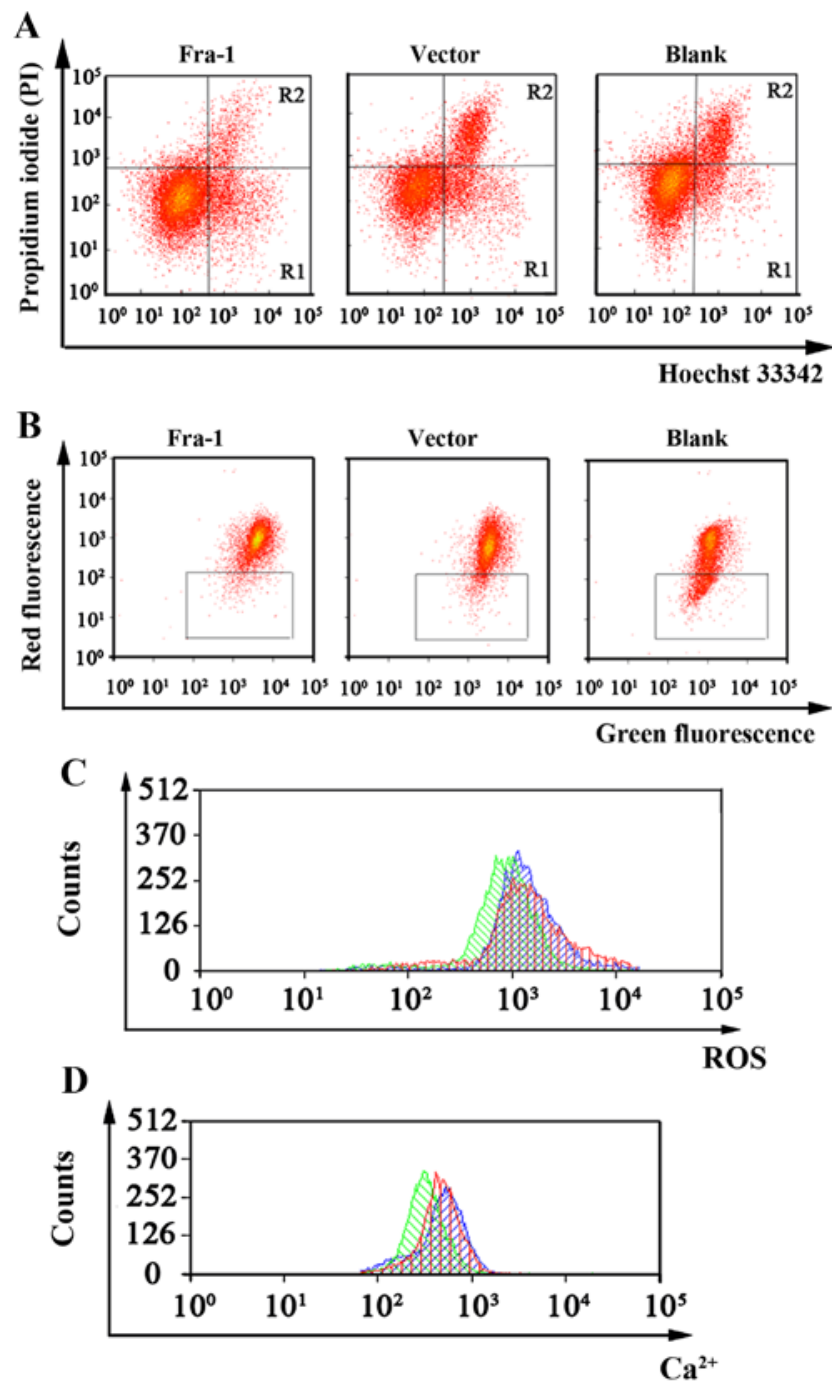

Figure 2. Assessment of cell apoptosis, mitochondrial membrane potential $(\Delta \Psi \mathrm{m})$, intracellular reactive oxygen species (ROS) and calcium ion $\mathrm{Ca}^{2+}$ concentration in H460, H460/vector and H460/Fra-1 cells. (A) Cell apoptosis of the H460, H460/vector and H460/Fra-1 cells was analyzed by flow cytometry. (B) $\Delta \Psi \mathrm{m}$ in the $\mathrm{H} 460, \mathrm{H} 460$ /vector and H460/Fra-1 cells was analyzed by flow cytometry. (C) ROS in the H460, H460/vector and H460/Fra-1 cells were analyzed by flow cytometry. Green indicates H460/Fra-1 cells, red indicates $\mathrm{H} 460 /$ vector cells, blue indicates $\mathrm{H} 460$ cells. (D) $\mathrm{Ca}^{2+}$ concentrations in the H460, H460/vector and H460/Fra-1 cells were assessed by flow cytometry. Green indicates H460/Fra-1 cells, red indicates H460/vector cells, blue indicates H460 cells. Data are representative of three independent experiments.

intracellular ROS (Fig. 2C). $\mathrm{Ca}^{2+}$ experimental results showed that overexpression of Fra-1 in lung cancer H460 cells, led to a

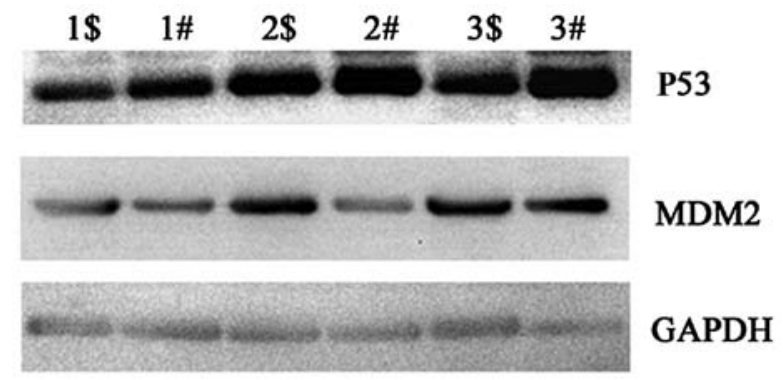

Figure 3. Expression levels of MDM2 and P53 protein in the lung cancer and adjacent non-cancerous tissues. In total, tissues (1-3) which were used in the detection of mRNA expression levels by qPCR were selected to detect the expression levels of MDM2 and P53 protein by western blot analysis. $1 \$-3 \$$, lung cancer tissues; $1 \#-3 \#$, the adjacent non-cancerous tissues. Data are representative of three independent experiments.

decrease in $\mathrm{Ca}^{2+}$ concentrations and suppressed the apoptosis of the lung cancer cells (Fig. 2D).

Fra-1 is correlated with dysregulation of the P53 signaling pathway in lung cancer tissues in vivo. To uncover the possible mechanism of Fra-1 in lung cancer, we tested the expression levels of key molecules in the P53 signaling pathway by western blot technology. P53 was downregulated in the lung cancer tissues compared with the level in the adjacent non-cancerous tissues, while MDM2 was upregulated in the lung cancer tissues (Fig. 3). Combined with the above result for which Fra-1 was upregulated in lung cancer, we propose that Fra-1 is correlated with dysregulation of the P53 signaling pathway in lung cancer tissues in vitro.

Fra-1 overexpression affects the expression of P53 and MDM2 in vitro. To confirm whether Fra-1 affects the expression of P53 and MDM2 in vivo, the H460 cells were transfected with the plasmid pEGFP-N1/Fra-1 or control vector. We harvested the cells and tested the expression levels of P53 and MDM2 proteins in vivo. P53 was downregulated in the $\mathrm{H} 460$ cells in which Fra-1 was overexpressed, while MDM2 was upregulated (Fig. 4). Our results revealed that Fra-1 overexpression affected the expression of P53 and MDM2 in vivo.

\section{Discussion}

Lung cancer is the leading cause of cancer-related death worldwide with a 5 -year survival rate of $<15 \%$, despite significant advances in both diagnostic and therapeutic approaches $(1,2)$. Lung cancer occurs and develops as a 


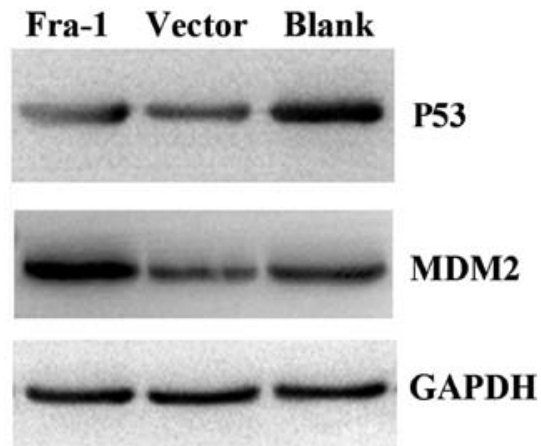

Figure 4. Expression levels of MDM2 and P53 protein in lung cancer cell lines. Fra-1, H460 cells transfected with pEGFP-N1-Fra-1. Vector, H460 cells transfected with pEGFP-N1. Blank, H460 cells which were not transfected with a plasmid. Data are representative of three independent experiments.

complicated result of an accumulation of various endogenous and exogenous effects. Gene alterations participate in lung cancer genesis (24-26). Thus, identifying molecular aberrations in lung cancer may improve our understanding of lung carcinogenesis and help us to subdivide patients into biologically and clinically relevant subgroups, as well as develop novel therapeutic strategies.

In this study, we found that the expression of the Fra-1 gene was higher in the lung cancer samples compared with that in the adjacent non-cancerous tissues and the normalized Fra-1 gene expression in lung cancer was upregulated by 3.76-fold. A similar trend was noted by western blot experiments. IHC showed a similar pattern in protein expression with the qPCR results and western blot results. A high score of Fra-1 was noted in $50.09 \%(13 / 22)$ of the lung cancer tissues and $13.64 \%(3 / 22)$ of the adjacent non-cancerous tissues. Motrich et al found that both Fra-1 and c-Fos were overexpressed in $>95 \%$ of human ductal breast carcinoma biopsies examined in contrast with very low or undetectable levels in normal tissue (27). Fra-1 is highly expressed in the muscle-invasive form of carcinoma of the bladder (28). Fos-related activator-1 is overexpressed in oral squamous cell carcinoma and is associated with tumor lymph node metastasis (29). Our results corresponded with the previous findings.

To uncover the potential mechanism of Fra-1 in lung cancer, we studied the effect of Fra-1 on the apoptosis of lung cancer H460 cells. Our results showed that there was a considerable decrease in apoptotic cells in the H460 cells with Fra-1 overexpession. Henckels and Prywes found that Fra-1 overexpression was sufficient to increase cell motility and anchorage-independent growth (30). High levels of Fra-1 in cells where elevated ERK activity leads to protein stabilization provide survival signals for tumor cells removed from the extracellular matrix (31). Fra-1 may influence the metastatic potential of breast cancer cells by altering the expression of adhesion molecules, resulting in increased adherence to endothelial cells under flow conditions (32). Collectively, the results suggested that Fra-1 may affect cell proliferation, invasion and apoptosis.

Cell apoptosis is closely related with a reduction in $\Delta \Psi \mathrm{m}$ and an increase in intracellular ROS and $\mathrm{Ca}^{2+}$ concentrations (33-38). In this study, we found that overexpression of
Fra-1 in lung cancer $H 460$ cells, led to an increase in $\Delta \Psi \mathrm{m}$ and a decrease in intracellular ROS and $\mathrm{Ca}^{2+}$ concentrations. Yang and Meyskens found that alterations in AP-1 transcription signaling, mediated by changes in AP-1 dimeric composition and reduced intracellular ROS levels, substantially contributed to the phenotypic changes induced by resveratrol (39). The rise in intracellular calcium concentration $\left(\mathrm{Ca}^{2+}\right)$ causes mitochondrial $\mathrm{Ca}^{2+}$ overload, thereby triggering apoptosis (40).

Furthermore, we found that Fra-1 was correlated with dysregulation of the P53 signaling pathway in lung cancer tissues in vitro, and Fra-1 overexpression affected the expression of MDM2 and P53 in vivo. Degradation of P53 is regulated by its interaction with specific E3 ubiquitin ligases, the best known one being encoded by MDM2 (41). Damage to the P53-dependent mechanism is often caused by overexpression of MDM2, which codes for a P53-regulating protein (42). Combined with the above results revealing that Fra-1 is highly expressed in lung cancer, we inferred that Fra-1 is upregulated in lung cancer tissues and inhibits the apoptosis of lung cancer cells by the P53 signaling pathway.

\section{Acknowledgements}

This study was supported by the National Natural Science Foundation of China (81272975, 81172302, 81402270 and 81402307); Key Project of Hunan Provincial Natural Science Foundation (12JJ2044); Project of the Hunan Provincial Natural Science Foundation (12JJ3121); Project of the Hunan Provincial Development and Reform Commission; the Planned Science and Technology Project of Hunan Province (2010FJ3088 and 2012FJ2014); and Hunan Provincial Innovation Foundation for Postgraduates.

\section{References}

1. Jemal A, Siegel R, Xu J and Ward E: Cancer statistics, 2010. CA Cancer J Clin 60: 277-300, 2010.

2. Wood SL, Pernemalm M, Crosbie PA and Whetton AD: Molecular histology of lung cancer: From targets to treatments. Cancer Treat Rev 41: 361-375, 2015.

3. Zhang F, Zhang X, Meng J, Zhao Y, Liu X, Liu Y, Wang Y, Li Y, Sun Y, Wang Z, et al: ING5 inhibits cancer aggressiveness via preventing EMT and is a potential prognostic biomarker for lung cancer. Oncotarget 6: 16239-16252, 2015.

4. Chen X, Song X, Yue W, Chen D, Yu J, Yao Z and Zhang L: Fibulin-5 inhibits Wnt/ $\beta$-catenin signaling in lung cancer. Oncotarget 6: 15022-15034, 2015.

5. Adiseshaiah P, Vaz M, Machireddy N, Kalvakolanu DV and Reddy SP: A Fra-1-dependent, matrix metalloproteinase driven EGFR activation promotes human lung epithelial cell motility and invasion. J Cell Physiol 216: 405-412, 2008.

6. Rege TA and Hagood JS: Thy-1 as a regulator of cell-cell and cell-matrix interactions in axon regeneration, apoptosis, adhesion, migration, cancer, and fibrosis. FASEB J 20: 10451054, 2006

7. Scognamiglio G,D'Antonio A, Rossi G,Cavazza A,Camerlingo R, Pirozzi G, La Mantia E, Anniciello AM, Morabito A, Cantile M, et al: CD90 expression in atypical meningiomas and meningioma metastasis. Am J Clin Pathol 141: 841-849, 2014.

8. Kitayama J, Emoto S, Yamaguchi H, Ishigami H, Yamashita H, Seto Y, Matsuzaki K and Watanabe T: CD90(+)CD45(-) intraperitoneal mesothelial-like cells inhibit $\mathrm{T}$ cell activation by production of arginase I. Cell Immunol 288: 8-14, 2014.

9. Ohmura-Kakutani H, Akiyama K, Maishi N, Ohga N, Hida Y, Kawamoto T, Iida J, Shindoh M, Tsuchiya K, Shinohara N, et al: Identification of tumor endothelial cells with high aldehyde dehydrogenase activity and a highly angiogenic phenotype. PLoS One 9: e113910, 2014. 
10. Ma K, Chang D, Gong M, Ding F, Luo A, Tian F, Liu Z and Wang T: Expression and significance of FRA-1 in non-small-cell lung cancer. Cancer Invest 27: 353-359, 2009.

11. Adiseshaiah P, Lindner DJ, Kalvakolanu DV and Reddy SP FRA-1 proto-oncogene induces lung epithelial cell invasion and anchorage-independent growth in vitro, but is insufficient to promote tumor growth in vivo. Cancer Res 67: 6204-6211, 2007.

12. Oikonomou D, Hassan K, Kaifi JT, Fiegel HC, Schurr PG, Reichelt U, Aridome K, Yekebas EF, Mann O, Kluth D, et al: Thy-1 as a potential novel diagnostic marker for gastrointestinal stromal tumors. J Cancer Res Clin Oncol 133: 951-955, 2007.

13. Jiang J, Zhang Y, Chuai S, Wang Z, Zheng D, Xu F, Zhang Y, Li C, Liang Y and Chen Z: Trastuzumab (herceptin) targets gastric cancer stem cells characterized by CD90 phenotype. Oncogene 31: 671-682, 2012

14. Goldar S, Khaniani MS, Derakhshan SM and Baradaran B Molecular mechanisms of apoptosis and roles in cancer development and treatment. Asian Pac J Cancer Prev 16: 2129-2144, 2015.

15. Paul I and Jones JM: Apoptosis block as a barrier to effective therapy in non small cell lung cancer. World J Clin Oncol 5: 588-594, 2014.

16. Beesoo R, Neergheen-Bhujun V, Bhagooli R and Bahorun T: Apoptosis inducing lead compounds isolated from marine organisms of potential relevance in cancer treatment. Mutat Res 768 : 84-97, 2014

17. Livak KJ and Schmittgen TD: Analysis of relative gene expression data using real-time quantitative PCR and the 2(-Delta Delta C(T)) method. Methods 25: 402-408, 2001.

18. Zhou Y, Wang W, Zheng D, Peng S, Xiong W, Ma J, Zeng Z, Wu M,Zhou M, Xiang J, et al: Risk of nasopharyngeal carcinoma associated with polymorphic lactotransferrin haplotypes. Med Oncol 29: 1456-1462, 2012

19. Xiao S, Zhou Y, Yi W, Luo G, Jiang B, Tian Q, Li Y and Xue M: Fra-1 is downregulated in cervical cancer tissues and promotes cervical cancer cell apoptosis by p53 signaling pathway in vitro. Int J Oncol 46: 1677-1684, 2015.

20. Zheng D, Liao S, Zhu G, Luo G, Xiao S, He J, Pei Z, Li G and Zhou Y: CD38 is a putative functional marker for side population cells in human nasopharyngeal carcinoma cell lines. Mol Carcinog: Jan 28, 2015 (Epub ahead of print). doi $10.1002 / \mathrm{mc} .22279$

21. Liao S, Xiao S, Zhu G, Zheng D, He J, Pei Z, Li G and Zhou Y: CD38 is highly expressed and affects the PI3K/Akt signaling pathway in cervical cancer. Oncol Rep 32: 2703-2709, 2014.

22. Zhu W, Li J, Su J, Li J, Li J, Deng B, Shi Q, Zhou Y and Chen X: FOS-like antigen 1 is highly expressed in human psoriasis tissues and promotes the growth of $\mathrm{HaCaT}$ cells in vitro. Mol Med Rep 10: 2489-2494, 2014

23. Hara A and Okayasu I: Cyclooxygenase- 2 and inducible nitric oxide synthase expression in human astrocytic gliomas: Correlation with angiogenesis and prognostic significance. Acta Neuropathol 108: 43-48, 2004.

24. Guo M, Alumkal J, Drachova T, Gao D, Marina SS, Jen J and Herman JG: CHFR methylation strongly correlates with methylation of DNA damage repair and apoptotic pathway genes in non-small cell lung cancer. Discov Med 19: 151-158, 2015.

25. Chiu CH, Ho HL, Doong H, Yeh YC, Chen MY, Chou TY and Tsai CM: MLH1 V384D polymorphism associates with poor response to EGFR tyrosine kinase inhibitors in patients with EGFR L858R-positive lung adenocarcinoma. Oncotarget 6 : 8407-8417, 2015

26. Van Nostrand JL, Brisac A, Mello SS, Jacobs SB, Luong R and Attardi LD: The p53 target gene SIVA enables non-small cell lung cancer development. Cancer Discov 5: 622-635, 2015.
27. Motrich RD, Castro GM and Caputto BL: Old players with a newly defined function: Fra-1 and c-Fos support growth of human malignant breast tumors by activating membrane biogenesis at the cytoplasm. PLoS One 8: e53211, 2013.

28. Sayan AE, Stanford R, Vickery R, Grigorenko E, Diesch J, Kulbicki K, Edwards R, Pal R, Greaves P, Jariel-Encontre I, et al: Fra-1 controls motility of bladder cancer cells via transcriptional upregulation of the receptor tyrosine kinase AXL. Oncogene 31: 1493-1503, 2012

29. Zhang L, Pan HY, Zhong LP, Wei KJ, Yang X, Li J, Shen GF and Zhang Z: Fos-related activator-1 is overexpressed in oral squamous cell carcinoma and associated with tumor lymph node metastasis. J Oral Pathol Med 39: 470-476, 2010.

30. Henckels E and Prywes R: Fra-1 regulation of matrix metallopeptidase-1 (MMP-1) in metastatic variants of MDA-MB-231 breast cancer cells. F1000Res 2: 229, 2013.

31. Vial E and Marshall CJ: Elevated ERK-MAP kinase activity protects the FOS family member FRA-1 against proteasomal degradation in colon carcinoma cells. J Cell Sci 116: 4957-4963, 2003.

32. Oliveira-Ferrer L, Kürschner M, Labitzky V, Wicklein D, Müller V, Lüers G, Schumacher U, Milde-Langosch K and Schröder C: Prognostic impact of transcription factor Fra-1 in ER-positive breast cancer: Contribution to a metastatic phenotype through modulation of tumor cell adhesive properties. J Cancer Res Clin Oncol 141: 1715-1726, 2015.

33. Hacker K and Medler KF: Mitochondrial calcium buffering contributes to the maintenance of Basal calcium levels in mouse taste cells. J Neurophysiol 100: 2177-2191, 2008.

34. Ogura T, Margolskee RF and Kinnamon SC: Taste receptor cell responses to the bitter stimulus denatonium involve $\mathrm{Ca}^{2+}$ influx via store-operated channels. J Neurophysiol 87: 3152-3155, 2002.

35. Akabas M, Dodd J and al-Awqati Q: Identification of electrophysiologically distinct subpopulations of rat taste cells. J Membr Biol 114: 71-78, 1990.

36. Pulkkinen V, Manson ML, Säfholm J, Adner M and Dahlén SE: The bitter taste receptor (TAS2R) agonists denatonium and chloroquine display distinct patterns of relaxation of the guinea pig trachea. Am J Physiol Lung Cell Mol Physiol 303: L956-L966, 2012.

37. Ruiz-Avila L, McLaughlin SK, Wildman D, McKinnon PJ, Robichon A, Spickofsky N and Margolskee RF: Coupling of bitter receptor to phosphodiesterase through transducin in taste receptor cells. Nature 376: 80-85, 1995.

38. Li Y, Su X, Wang X, Leung AW, Xu C, Wang P and Liu Q: Cytotoxic effect of protoporphyrin IX to human leukemia U937 cells under ultrasonic irradiation. Cell Physiol Biochem 33: 1186-1196, 2014

39. Yang S and Meyskens FL Jr: Alterations in activating protein 1 composition correlate with phenotypic differentiation changes induced by resveratrol in human melanoma. Mol Pharmacol 67: 298-308, 2005

40. Liu KH, Yang ST, Lin YK, Lin JW, Lee YH, Wang JY, Hu CJ, Lin EY, Chen SM, Then CK, et al: Fluoxetine, an antidepressant, suppresses glioblastoma by evoking AMPAR-mediated calcium-dependent apoptosis. Oncotarget 6: 5088-5101, 2015.

41. Chumakov PM: Function of the p53 gene: Choice between life and death. Biochemistry (Mosc) 65: 28-40, 2000.

42. Chipuk JE and Green DR: Dissecting p53-dependent apoptosis. Cell Death Differ 13: 994-1002, 2006. 